\title{
Medical Abortion in Family Practice: A Case Series
}

\author{
Linda Prine, MD, Ruth Lesnewski, MD, Nancy Berley, and Marji Gold, MD
}

Background: We wanted to determine the outcomes of medical abortions in four family practice centers.

Methods: This study was a retrospective case series of consecutive medical abortions in four community health centers between November 2000 and April 2002. We defined a successful medical abortion as one that required no further intervention after the administration of the medications mifepristone and misoprostol. The subset of abortions in patients who had suction procedures were called failures.

Results: In this series of 236 abortions, only 1 woman had a viable pregnancy after taking the medication as directed, and she had an elective suction procedure to terminate the pregnancy. None of the patients under the complete care of family physicians received suction procedures for other indications. Two patients underwent suction procedures at other institutions for unknown indications. Eight were lost to follow-up. One did not adhere to the protocol and so was excluded from the data analysis. The failure rate of the protocol for patients cared for by the family physicians at follow-up was $\mathbf{0 . 4 \%}$.

Conclusions: Medical abortion in a family practice setting is a safe and effective procedure. If practiced widely, it could make abortion care much more accessible to women. (J Am Board Fam Pract 2003;16:290-5.)

Mifepristone was used in Europe and then Asia for medical abortion for almost a decade before it was submitted to the Food and Drug Administration for approval in $1996 .{ }^{1-5}$ Before the final approval for commercial distribution in September 2000, several trials were done in the United States. ${ }^{6-8}$ Since the release of mifepristone, more studies have been published establishing its efficacy and safety. ${ }^{9-11}$ These large, multicenter trials compiled data from a range of settings, including university hospitals, abortion clinics, private gynecology practices, and family medicine offices. Although these studies included family medicine community health centers, the data were not reported in a way that allowed us to evaluate the safety and efficacy of offering medical abortions in a primary care, community setting.

Because $45 \%$ of women of reproductive age in the United States have at least one abortion, and most are done at less than 8 weeks' gestational age, ${ }^{12}$ it is important to evaluate the safety of offering medical abortions in these community settings. Also, given that $86 \%$ of all counties in the United States have no abortion provider, ${ }^{12}$ medical

Submitted, revised, 9 August 2002.

From the Beth Israel Residency in Urban Family Practice (LP, RL), and the Albert Einstein College of Medicine (NB, MG), New York. Address reprints to Linda Prine, MD, Beth Israel Residency in Urban Family Practice, $16 \mathrm{E} 16^{\text {th }}$ Street, New York, NY 10003. abortion in family practice settings could greatly expand the availability of this aspect of women's reproductive health care. Most suction abortions $(55 \%)$ occur at less than 8 weeks of gestational age, ${ }^{12}$ which puts them in the time frame of eligibility for a medical abortion.

Early mifepristone research trials defined a successful medical abortion as one that required no further intervention after the administration of the medications mifepristone and misoprostol, and failure as one that resulted in continued pregnancy or suction intervention for any cause. These early studies reported success rates of more than $90 \%,{ }^{13}$ and rates in later trials reached higher than $95 \% .{ }^{8,9}$ The most successful studies used $200 \mathrm{mg}$ of mifepristone orally and $800 \mu \mathrm{g}$ of misoprostol vaginally. ${ }^{11}$ With this regimen, the rate of failed medical abortions was less than $3 \%$. $^{9}$

To date, no published trials have focused on the outcomes of medical abortion provided in dedicated family practice settings. Because the long delay in approval of the medication was attributed partially to concerns for its safety in community settings, examining outcomes of medical abortion in primary care offices is extremely important. Additionally, health insurance companies have been slow to develop policy on reimbursement of family physicians for medical abortions (see: www. earlyoptionpill.com). Because medical abortion involves counseling and medication administration 
rather than an invasive procedure, medical abortion is appropriate for a primary care setting and should be reimbursed at a rate equivalent to similar office visits. The purpose of this article is to examine the outcomes of medical abortions in four family practices and discuss the outcomes in comparison to previous published studies.

\section{Methods}

We reviewed charts to collect information about all consecutive medical abortions performed in four urban family practice clinics between November 2000 and April 2002.

We kept a $\log$ of the mifepristone lot numbers and the patient chart numbers. As part of the regular quality improvement process, we abstracted the data from charts. Our chart review collected information on patient age, insurance (as a proxy for economic status), gestational age, whether the patient had a sonogram, and outcome. All patients who visited these four family practices for medical abortions and who completed the clinical protocol as prescribed are included in this study. If the patient did not take the second medication, or if she took it immediately upon arriving at home instead of waiting 24 to 72 hours, she was excluded from the study. Women who did not keep their follow-up appointments to assess the completion of their abortion, but who were contacted by telephone and gave a history that allowed the provider to conclude the abortion was complete, were included. If the history seemed inconclusive, the women were strongly encouraged to come to the center for an assessment.

The four family practice sites provided 236 medical abortions to 233 women. Of the 236 cases, 8 were excluded from the analysis of the success rate because the women did not return for follow-up and could not be reached by telephone. One additional patient was excluded for failing to follow the protocol—she inserted her misoprostol on the same day she took the mifepristone. Only the 227 women who returned or who gave information by telephone and adhered to the protocol are included in the analysis. Follow-up was obtained on a rolling basis: patients who did not keep their second appointment were telephoned and asked to come in. If they still did not return for the second visit, they were telephoned again, and a history was taken by telephone. Some patients re-
Table 1. Patient Characteristics.

\begin{tabular}{lrr}
\hline Characteristic & Number & Percent \\
\hline Age, years & & \\
16-21 & 39 & 17 \\
$22-29$ & 102 & 45 \\
30-39 & 77 & 34 \\
40+ & 9 & 4 \\
Total & 227 & 100 \\
Types of insurance & & \\
Medicaid & 53 & 24 \\
Private Insurance & 89 & 39 \\
Uninsured/Self Pay & 80 & 35 \\
Unknown & 5 & 2 \\
Total & 227 & 100 \\
Gestational age & & \\
$\quad<6$ wk & 66 & 29 \\
6 wk-6 wk 6 d & 67 & 30 \\
7 wk-7 wk 6 d & 63 & 28 \\
8-9 wk & 31 & 13 \\
Total & 227 & \\
\hline
\end{tabular}

turned months later for other health needs, often for other family members, and their outcomes were confirmed at that time.

\section{Setting}

Physicians worked in clinical family medicine sites in Manhattan and the Bronx, NY. All practices served an ethnically diverse, primarily low-income population. As displayed in Table 1, most patients had Medicaid or were uninsured. Family physicians, residents, and advanced practice clinicians (nurse practitioners and physician's assistants) staffed the family practices.

\section{Description of the Clinical Protocol}

The clinical protocol we used was based on the Abortion Rights Mobilization (ARM) trials (Table 2). The provider administered $200 \mathrm{mg}$ of oral mifepristone in the office, and the patient self-administered $800 \mu \mathrm{g}$ of vaginal misoprostol at home 24 to 72 hours later. ${ }^{7-9}$ The gestational age limit was 63 days. Thus, our clinical protocol differed from the FDA-approved product labeling, which specifies a mifepristone dosage of $600 \mathrm{mg}, 400 \mu \mathrm{g}$ of oral misoprostol taken in the office 48 hours later, and a gestational age limit of 49 days. We chose to follow the ARM protocol for its greater efficacy, ${ }^{11}$ enhanced privacy and flexibility, and lower cost.

We confirmed pregnancies by urine testing and either serum human chorionic gonadotropin level (hCG) or vaginal sonogram. The process began with the primary care clinician doing the initial options counseling, reviewing the procedure with 


\begin{tabular}{|c|c|c|}
\hline & ARM Protocol & FDA Product Labeling \\
\hline Gestational age limit & 63 days & 49 days \\
\hline Mifepristone dose & $200 \mathrm{mg}$ orally & $600 \mathrm{mg}$ orally \\
\hline Misoprostol dosing & $\begin{array}{l}800 \mu g \text { vaginally } \\
\text { Home self-administration } \\
24-72 \text { h later (day } 1-3)\end{array}$ & $\begin{array}{l}400 \mu g \text { orally } \\
\text { Office administration } \\
48 \text { h later (day 2) }\end{array}$ \\
\hline Office follow-up visit & day 4-8 & day $10-15$ \\
\hline Minimum office visits & 2 & 3 \\
\hline Cost & $\begin{array}{l}\$ 90 \text { for mifepristone } \\
2 \text { office visits } \\
\$ 4 \text { for misoprostol }\end{array}$ & $\begin{array}{l}\$ 270 \text { for mifepristone } \\
3 \text { office visits } \\
\$ 2 \text { for misoprostol }\end{array}$ \\
\hline
\end{tabular}

ARM — Abortion Rights Mobilization trials, FDA — Food and Drug Administration.

the patient, obtaining informed consent, and administering the mifepristone. This physician was available by beeper to the patient until the follow-up visit.

Medical abortion success was defined as completion of the abortion using only the medications mifepristone and misoprostol, confirmed by history and either a serum hCG decline of more than $50 \%$, or a sonogram showing the absence of a viable pregnancy. Success was additionally defined as completing this process without need for a surgical intervention (a suction evacuation of the uterus). Indications for suction evacuation included acute and prolonged heavy vaginal bleeding, incomplete abortion (retained products of conception), or continuing pregnancy. A repeated dose of misoprostol was offered as an alternative if there were sonographic findings of persistent heterogeneous, echogenic material without evidence of a continued viable pregnancy.

The decision about whether to do a sonogram for dating of the pregnancy before the administration of mifepristone depended on several factors. Indications for mandatory preabortion sonograms included uncertain date of last menstrual period, size and date discrepancy at the initial examination, pregnancy dated in the 9th week, and a history of taking hormonal contraceptives at the time of the patient's last menstrual period. Indications for mandatory postabortion sonograms included a serum hCG level that had not decreased by at least $50 \%$ and a history consistent with an incomplete abortion, such as no cramps or bleeding or persistent symptoms of pregnancy.

For most patients, sonograms were done routinely without documentation of indications. With time, some providers became comfortable evaluat- ing the need for sonography as described above, and fewer patients had routine sonograms. Sonography was performed for 205 patients and was deemed unnecessary in 22 women who had abortions toward the end of the series.

In offices where the family physician could not perform a suction procedure, backup arrangements had been made with other physicians who worked in clinics or hospitals where suction abortions could be performed.

\section{Results}

Only the 227 cases of women who adhered to the protocol and for whom we had complete follow-up information were included (Table 3). Two hundred twenty-five of these cases were considered successful medical abortions. The women who did not return for a second visit, but who by telephone expressed certainty that the procedure had been successful and described cramping and bleeding and the disappearance of symptoms of pregnancy, were included. No women who had not returned but was contacted by telephone gave a history of a continuing pregnancy or any history other than that described above.

One woman underwent a suction procedure for medication failure. She was $61 / 2$ week's pregnant at the first visit, returned in 1 week, and had a sonogram showing a viable 7 1/2-week pregnancy. She did report to her provider before the sonogram that she still felt pregnant and that she had not bled very much. Follow-up examination after the suction procedure showed the abortion to be complete. Another patient reported by telephone that she went to an emergency department in another state and underwent a dilatation and curettage. 


\begin{tabular}{lrr}
\hline Criteria & Number & Comments \\
\hline $\begin{array}{l}\text { Total number of medical abortions } \\
\begin{array}{l}\text { Excluded because they did not return and were unable to } \\
\text { be contacted for follow-up examination }\end{array}\end{array}$ & $\begin{array}{r}236 \\
\text { Excluded for failure to adhere to protocol }\end{array}$ & $\begin{array}{c}\text { These patients might return, as several patients did } \\
\text { months later }\end{array}$ \\
$\begin{array}{l}\text { Patients who adhered to the protocol and had complete } \\
\text { follow-up care }\end{array}$ & $\begin{array}{c}227 \\
\text { Genuine method failures }\end{array}$ & $\begin{array}{c}\text { This patient underwent a suction procedure for unknown } \\
\text { indications }\end{array}$ \\
$\begin{array}{l}\text { 226 had a successful medical abortion using mifepristone } \\
\text { and misoprostol according to study protocol }\end{array}$ & 1 & $\begin{array}{c}\text { This patient had a continuing pregnancy and required a } \\
\text { suction procedure to complete her abortion }\end{array}$ \\
\hline
\end{tabular}

Of the 227 cases that were included, only one received a second dose of misoprostol because of a persistent, although abnormal shaped, gestational sac observed on the sonogram at the follow-up visit. The woman was assessed by sonogram again 1 week later and had an empty uterus. Three other patients were offered, but declined, a second dose of misoprostol for heterogeneous echogenic material on sonography and prolonged bleeding without a decline in hematocrit. Their bleeding stopped after their subsequent menstrual cycle. All other women completed the medical abortion with the initial doses only.

One patient was excluded because she mistakenly inserted the misoprostol only a few hours after the mifepristone and then did not return for her follow-up visit. At a visit months later (when she came in for another medical abortion), she reported to us that she had returned to a gynecology practice where she underwent a dilatation and curettage. We were unable to obtain her medical records.

Eight cases were lost to follow-up, including 1 woman who told us by telephone that she never inserted the misoprostol and was then lost to follow-up.

Because none of the women who returned for follow-up care needed a surgical procedure (suction curettage) for any indication other than the one continuing pregnancy, the failure rate was 1 of 227 , or $0.4 \%$ (95\% CI, 0.24-7.22). By including the patient who did not return but went to an emergency department in another state and received a dilatation and curettage for unclear indications, our failure rate becomes $0.8 \%$. It is possible that other women who were lost to follow-up underwent suction procedures in emergency departments. If we were to assume that all our patients who did not return for follow-up required intervention for failed abortions and included them in our numbers, our failure rate would become 11 out of 236 , or $4.7 \%$ (95\% CI, 5.49-19.68). Because all but 8 of our patients who were lost to follow-up eventually returned to us for another problem and then reported a successful abortion, however, the true failure rate is probably closer to $0.4 \%$.

\section{Discussion}

This series shows a very high rate of success and minimal complications of medical abortion in a family practice setting. In our chart review of 227 patients with known outcomes who adhered to the protocol, 99.2\% successfully completed a medical abortion with the oral administration of mifepristone and then home administration of vaginal misoprostol. Only 1 patient had a true medication failure requiring a suction procedure. Among the patients who returned to us for follow-up care, none required a suction procedure for complications (prolonged heavy bleeding, retained tissue, hemorrhage).

Most women in this series either had Medicaid or were uninsured. Our high rate of patients returning for follow-up care (96\%) shows the enhanced capacity of family physicians in continuity care practices to monitor patients through the medical abortion process. At a family planningabortion clinic in a major metropolitan area where statistics on follow-up for medical abortion were kept, the return for follow-up rate was $75 \%$, and in a smaller clinic the follow-up rate was $82 \%$ (unpublished data from Planned Parenthood Federation of America).

Although data show that hemorrhage during the acute phase of a medical abortion is rare,${ }^{14}$ many of 
the women in our series might have received surgical intervention had they gone to emergency departments for bleeding during their medical abortion process, as we saw with the patient who did not call us before going to an emergency department. Because all patients had been counseled to telephone their physician for any worrisome symptoms, the physicians were able to assess the bleeding and reassure the women.

There are only two absolute indications for surgical intervention after a medical abortion: a continuing, viable pregnancy, and serious hemorrhage. The reported rate of actual continuing pregnancy after medical abortion with this regimen is $0.4 \%,{ }^{10}$ which matches our experience. Hemorrhage requiring transfusion usually occurs several weeks after the initial phase of bleeding, often after an uneventful follow-up examination. ${ }^{15}$ Because the incidence of bleeding requiring transfusion is much lower than 1 in $200,{ }^{16}$ we might not have reached this number because we had not yet accumulated a sufficient number of patients. The rate for suction procedures for any indication in the previously published US studies was 3\% to 5\%. Even if all our patients lost to follow-up actually went to emergency departments and received suction procedures, our failure rate would still be only $4.7 \%$ (11 of 236). The authors of the large studies we referenced did not report whether their suction procedures occurred in emergency departments or in the practices doing the studies.

The published studies from the United States have all used sonography before and after abortion. It is possible that the postabortion sonogram contributes to the higher rate of suction procedures. The literature does show that with experience there is a decline in the rate of suction procedures after medical abortion, as physicians become accustomed to finding diffuse heterogeneous echogenic material along the endometrial stripe after a medical abortion. ${ }^{17}$ Another explanation might be that the physicians in the research trials were also providers of surgical abortion and thus more comfortable with suction procedures. Generally, family physicians provide expectant management of spontaneous abortion more often than gynecologists, with comparable, safe outcomes, ${ }^{18,19}$ and so we would expect this comfort with expectant management to apply to medical abortion as well.

For most of the women in the study, sonography was the main test used to confirm gestational age and outcome of the procedure. Although this series illustrates the feasibility of using sonography in family practices, we also could see that it was not necessary to use sonography routinely or to have it on site. Although we believe, based on our experience, that sonography is not necessary in most cases, it would be helpful to have more studies and reports of this sonogram-as-needed regimen. Studies of medical abortion in both less developed countries and Europe have comparably low complication rates where sonography is not available for routine use. ${ }^{20,21}$

\section{Conclusion}

Our data support the feasibility and appropriateness of providing medical abortion within family medicine settings. As first trimester miscarriages can be managed expectantly without intervention, the great majority of cases of medical abortion can be completed without need for surgical intervention.

Medical abortion in the primary care office is a safe and effective alternative to suction procedures, giving family physicians and advanced practice clinicians the opportunity to provide women with a fuller scope of reproductive choices. In the rare case in which an ongoing pregnancy requires a suction procedure, it can be scheduled electively at an appropriate facility. Family physicians can integrate counseling, history and physical examination, mifepristone administration, and follow-up into routine visits. The high compliance with follow-up visits in continuity practice, primary care settings provides additional evidence of safety.

\section{References}

1. Termination of pregnancy with reduced doses of mifepristone. World Health Organization Task Force on Post-ovulatory Methods of Fertility Regulation. BMJ 1993;307:532-7.

2. Sitruk-Ware R, Thalabard JC, De Plunkett TL, et al. The use of the antiprogestin RU486 (mifepristone) as an abortifacient in early pregnancy-clinical and pathological findings; predictive factors for efficacy. Contraception 1990;41:221-43.

3. Ashok PW, Penney GC, Flett GM, Templeton A. An effective regimen for early medical abortion: a report of 2000 consecutive cases. Human Reprod 1998;13:2962-5.

4. Winikoff B, Sivin I, Coyaji K, et al. Safety, efficacy, and acceptability of medical abortion in China, Cuba, and India: a comparative trial of mifepristone- 
misoprostol versus surgical abortion. Am J Obstet Gynecol 1997;176:431-7.

5. Winikoff B, Sivin I, Coyaji K, et al. The acceptability of medical abortion in China, Cuba and India. Int Fam Plann Perspect 1997;23:73-8.

6. Winikoff B, Ellertson C, Elul B, Sivin I. Acceptability and feasibility of early pregnancy termination by mifepristone-misoprostol. Results of a large multicenter trial in the United States. Mifepristone Clinical Trials Group. Arch Fam Med 1998;7:360-6.

7. Schaff EA, Stadalius LS, Eisinger SH, Franks P. Vaginal misoprostol administered at home after mifepristone (RU486) for abortion. J Fam Pract 1997;44:353-60.

8. Schaff EA, Eisinger SH, Stadalius LS, Franks P, Gore BZ, Poppema S. Low-dose mifepristone 200 $\mathrm{mg}$ and vaginal misoprostol for abortion. Contraception 1999;59:1-6.

9. Schaff EA, Fielding SL, Westhoff C, et al. Vaginal misoprostol administered 1,2 , or 3 days after mifepristone for early medical abortion: a randomized trial. JAMA 2000;284:1948-53.

10. Allen RH, Westhoff C, De Nonno L, Fielding SL, Schaff EA. Curettage after mifepristone-induced abortion: frequency, timing, and indications. Obstet Gynecol 2001;98:101-6.

11. Schaff EA, Fielding SL. A comparison of the abortion rights mobilization and population council trials. J Am Med Womens Assoc 2000;55(3 Suppl): 137-40.

12. Henshaw SK. Abortion incidence and services in the United States, 1995-1996. Fam Plann Perspect 1998;30:263-70, 287.

13. Newhall EP, Winikoff B. Abortion with mifepristone and misoprostol: regimens, efficacy, acceptabil- ity and future directions. Am J Obstet Gynecol 2000; 183(2 Suppl):S44-53.

14. Davis A, Westhoff C, De Nonno L. Bleeding patterns after early abortion with mifepristone and misoprostol or manual vacuum aspiration. J Am Med Womens Assoc 2000;55(3 Suppl):141-4.

15. Harper C, Winikoff B, Ellerston C, Coyaji K. Blood loss with mifepristone-misoprostol abortion: measures from a trial in China, Cuba and India. Int J Gynaecol Obstet 1998;63:39-49.

16. Kruse B, Poppema S, Creinin MD, Paul M. Management of side effects and complications in medical abortion. Am J Obstet Gynecol 2000;183(2 Suppl): S68-75.

17. Borgatta L, Burnhill M, Haskell S, Nichols M, Leonhardt, K Instituting medical abortion services: changes in outcome and acceptability related to provider experience. J Am Med Womens Assoc 2000; 55(3 Suppl):173-6.

18. Geyman JP, Oliver LM, Sullivan SD. Expectant, medical, or surgical treatment of spontaneous abortion in first trimester of pregnancy? A pooled quantitative literature evaluation. J Am Board Fam Pract 1999; 12:55-64.

19. Nielsen S, Hahlin M, Platz-Christensen J. Randomised trial comparing expectant with medical management for first trimester miscarriages. Br J Obstet Gynaecol 1999;106:804-7.

20. Coyaji K. Early medical abortion in India: three studies and their implications for abortion services. J Am Med Womens Assoc 2000;55(3 Suppl):191-4.

21. Elul B, Hajri S, Ngoc NN, et al. Can women in less-developed countries use a simplified medical abortion regimen? Lancet 2001;5:357:1402-5. 